\title{
Group A2 Analogs of Innovator Teicoplanin: Variability of their Ratio during Treatment Time Course in Human Blood; Consequences for the Generics
}

\author{
Jehl $F^{1 *}$, Schramm $F^{1}$, Colombo $P^{2}$ and Brink $A^{3}$ \\ ${ }^{1}$ Laboratory of Bacteriology, Department of Microbiology, University Hospital of Strasbourg, Strasbourg, France \\ ${ }^{2}$ Department of Pharmacy, University of Parma, Parma, Italy \\ ${ }^{3}$ Department of Clinical Microbiology, Milpark Hospital, Johannesbourg, South Africa
}

\begin{abstract}
Absence of therapeutic equivalence (TE) of generic antibiotics versus the innovator has been reported, even in case of pharmaceutical equivalence (PE).The pharmaceutically active principle of teicoplanin is mainly represented by the A2 group analogs (A2-1 to A2-2) which exist in given ratios in the innovator.

We studied the evolution of these ratios in humans volunteers receiving 2 dosage regimen $(6 \mathrm{mg} / \mathrm{kg}$ IV every 12 hours, 3 times, and then $6 \mathrm{mg} / \mathrm{kg}$ per day for ten days - group1-, and $12 \mathrm{mg} / \mathrm{kg}$ IV every $12 \mathrm{~h}$, 3 times, followed by 15 $\mathrm{mg} / \mathrm{kg}$ every other day till D11 - group 2-), by a specific HPLC methodology, after single dose (D1) and at steady state at day 11(D11).

The less lipophilic analogs $\mathrm{A} 2-1, \mathrm{~A} 2-2$, and A2-3 percentage decreases respectively were $18 \%, 11 \%$, and $21 \%$ (group 1) and $34 \%, 14 \%$, and $15 \%$ (group 2) between D1 and D11 for trough concentrations. At the same time, the more lipophilic A2-4 and A2-5 analogs ratio increases respectively were $15 \%$ and $19 \%$ (group 1 ) and $13 \%$ and $30 \%$ (group 2). These variations raise the problem of generics having the same global qualitative composition of analogs (PE) but with differing initial ratios. The bactericidal activity of innovator teicoplanin is optimal at its own ratios of the different analogs that act in a synergistic manner. Regarding generics, it would lead to final ratio in blood and tissues that are at risk of a sub optimal bactericidal activity. Thus, concluding in bioequivalence and therapeutic equivalence of these generics with the innovator remains highly questionable.
\end{abstract}

Keywords: Teicoplanin; A2 analogs; innovator; generics; therapeutic equivalence

\section{Introduction}

An increasing number of recently published papers report the absence of Therapeutic Equivalence (TE) of generic antibiotics when compared with the innovator, even in case of Pharmaceutical Equivalence (PE). On the basis of in vitro $\mathrm{PK} / \mathrm{PD}$ data or in vivo experimental infections data, generics of antibiotics such as gentamicin, oxacillin, vancomycin and more recently teicoplanin have been reported to present risks of failure of therapeutic equivalence although PE was proven [1-5]. Indeed, according to the official WHO definition [6], an antibiotic drug, to be considered as a generic, must, at first, exhibit the same qualitative and quantitative composition of active substance than the innovator. Then the bioequivalence must be proven by either an equivalent bioavailability or equivalent therapeutic effect between the generic and the innovator. This is also stated by the FDA and EMEA [7], for who TE results from PE plus bioequivalence. By definition, for intravenous drugs, the bioequivalence is always reached as far as the bioavailability is considered to be $100 \%$ as no absorption or resorption processes are involved. Nevertheless, one has to keep in mind that, even with IV drugs, a $100 \%$ bioavailability does not necessarily imply bioequivalence: a short duration IV bolus may result in the same area under the curve (AUC) than a $1 \mathrm{~h}$ infusion of the same amount, but accompanied with a higher peak concentration, what may result in a different efficacy for concentration- dependant antibiotics. Currently, because two IV forms are considered bioequivalent, the assessment of TE of generics when compared with innovator only depends of the quality of the raw materials used in the generic [6,7].

Teicoplanin is an injectable antibiotic produced by fermentation of Actinoplanes teichomyceticus. The pharmaceutically active principle is teicoplanin A2, a complex mixture of compounds designated A2-1 to A2-5, all differing in their length and branching of the fatty acid moiety linked to the glucosamine residue. They account for $90-95 \%$ of the total teicoplanin complex. A sixth more polar active component, teicoplanin $\mathrm{A} 3$, is always present in the final product, but does not exist in the fermentation broth. It has been identified as a degradation product of teicoplanin A2. It has been recently shown [8] that the production of the individual factors A2-2 to A 2-5 can be deeply influenced by the composition of the fermentation medium. The addition in the medium of precursors such as methyl linoleate, trilinoleate and crude cottonseed and/or corn oils can enhance the production of A2-1. In the same way, addition of methyl oleate, trioleate and olive and/or lard oils can stimulate the production of A2-3. The percentage of A2-2, A2-4 and A2-5 are significantly increased by the addition of amino acids such as $\mathrm{L}$-leucine, $\mathrm{L}$-isoleucine and $\mathrm{L}$-valine. It results that the final composition of teicoplanin in terms of the proportion of each component is deeply influenced by the fermentation medium composition and the production process. It is thus logical to consider that, depending on the manufacturing process; the five major components of teicoplanin may have different quantitative distribution in the current generics. The European Pharmacopoeia monograph for teicoplanin [9] gives

*Corresponding author: Jehl F, jhel Laboratory of Bacteriology, Department of Microbiology, University Hospital of Strasbourg, France, Tel: 0033 (0)3 69 551454 (hospital), 0033 ( 0) 368853790; Fax: 0033 (0)3 688538 08; E-mail: mailto:jehl@unistra.fr

Received August 20, 2013; Accepted August 21, 2013; Published September 20, 2013

Citation: Jehl F, Schramm F, Colombo P, Brink AJ (2013) Group A2 Analogs of Innovator Teicoplanin: Variability of their Ratio during Treatment Time Course in Human Blood; Consequences for the Generics. Pharmaceut Reg Affairs 2: 112. doi:10.4172/2167-7689.1000112

Copyright: @ $2013 \mathrm{Jehl} F$, et al. This is an open-access article distributed under the terms of the Creative Commons Attribution License, which permits unrestricted use, distribution, and reproduction in any medium, provided the original author and source are credited. 
the following limits for the acceptability of the different components ratio: $35-55 \%$ for A2-2, and an undifferenciated upper limit of no more than $20 \%$ for the other components. Colombo et al. [10] compared the composition of eleven batches of the innovator (Targocid/Targosid) with fourteen generics from different manufacturer. They concluded to statistically significant differences for Teicoplanin A2-1 (A2-1), A2-2, A2-3, A2-4 and A2-5 between some of the generics and the innovators. The distribution of the components of the generics unpredictably changed with the manufacturer. This is a concern for patients: Hanada et al. [11] showed that the six analogs of teicoplanin have different activities against specific bacterial species, and due to their different chemical structure, the different analogs have variable lipophilicity resulting in variable protein binding and pharmacokinetic behavior. Bernaregi et al. [12] concluded to a correlation between lipophilicity and decrease of some PK parameters such as total and renal clearances. They predicted a more pronounced accumulation of the more lipophilic compounds A2-4 and TA2-5 versus the others.

In a study published a few years ago [13], we investigated the trough and peaks concentrations of teicoplanin in volunteers at single dose and at steady state, after the administration of two dosage regimen: $6 \mathrm{mg} /$ $\mathrm{kg} / 24 \mathrm{~h}$ and $15 \mathrm{mg} / \mathrm{kg} / 48 \mathrm{~h}$. Only the total teicoplanin concentrations measured by fluorescence polarization immunoassay (FPIA) were published and used for PK parameters calculation. However, all the serum concentrations for the whole study were also measured by high performance liquid chromatography which allowed the measurement of all the A2 group analogs individually (A2-1 to A2-5).

We present here the results of the evolution, during this study, of the five compounds and their respective ratio at trough and peak concentrations at day 1 (single dose) and day 11 (steady state).

\section{Study Subjects and Procedure}

The study was a single-center, randomized, open-label, parallel group, control trial including 24 volunteers [13]. Two treatment groups were developed. Group 1 received $6 \mathrm{mg} / \mathrm{kg}$ intravenously every 12 hours 3 times and then $6 \mathrm{mg} / \mathrm{kg}$ per day from day 3 to 10 , and group 2 received $12 \mathrm{mg} / \mathrm{kg}$ intravenously every 12 hours three times and then $15 \mathrm{mg} / \mathrm{kg}$ every other day on days 3, 5, 7 and 9 .

\section{Teicoplanin Dosing and Sampling Times}

Four hundred mg freeze dried teicoplanin-containing vials (batch A0007; Gruppo Lepetit, Anagni, Italy) with a 24-mg sodium chloride excipient that had been stored at room temperature for a maximum of 3 months were reconstituted with $3.2 \mathrm{ml}$ of sterile water just before each infusion. A strictly weight-adjusted dose was given by a direct manual 1-min infusion in the ante-cubital vein.

The concentrations in the sera of subjects in groups 1 and 2 were measured at $1 \mathrm{~h}$ post-dosing except the second infusion on day $1(12 \mathrm{~h})$. Trough concentrations in serum were measured before the last infusion on the loading dose period $(24 \mathrm{~h})$ and then before each administration until day 10 (group 1) or day 9 (group 2). In addition, in group 2, mid-dose concentrations in serum were measured 24 hours after the administration of each dose on every day from day 4 to day 9. Thus a mid-dose concentration and a trough concentration $(48 \mathrm{~h}$ after administration of the last dose) were measured for the subjects in group 2 after each $15 \mathrm{mg} / \mathrm{kg}$ infusion.

\section{Analytical Procedure}

The samples were collected in dry vials without anticoagulant, allowed to clot at room temperature for $20 \mathrm{~min}$, and then centrifuged at
$2500 \times \mathrm{g}$ at $4^{\circ} \mathrm{C}$. The resulting serum sample was transferred to another tube for storage at $-80^{\circ} \mathrm{C}$ until assay.

Concentrations in serum were measured by high performance liquid chromatography according to a previously published method [14]. All compounds of the A2 group, A2-1 to A2-5, were measured in each sample allowing thus to calculate the proportion of each analog for each sample. All samples of the study have been already measured by a fluorescence polarization immunoassay (FPIA) and the results published elsewhere [13] in terms of a randomized comparison of serum teicoplanin concentrations following the daily and the alternate daily dosing [13]. For some specific sampling times, such as peaks and troughs, mean serum concentrations as measured by HPLC and FPIA were compared using the Student t-test for comparison of the means [15]. A p value $<0.01$ was considered as significant.

\section{Results}

\section{Serum HPLC and FPIA concentrations}

Peak and trough concentrations as measured by HPLC and FPIA [13] are shown in Table 1. All values as measured by FPIA are systematically superior to those measured by HPLC. Nevertheless for some of them, the difference is not significant whereas it is significant for others such as last peak concentrations in group 1 and group 2, and last trough concentration in group 2 . The difference is not statistically significant for trough concentrations in group 1 (Table 1).

Ratio of different components of the A2 group between day 1 (D1) and D11, for peaks as well as for trough concentrations, it can be observed that the proportions of A2-1, A2-2 and A2-3 decrease, when those of A2-4 and A2-5 increase (Tables 2 and 3). The most pronounced differences are those related to A2-1 and A2-5. Thus, for residual values in group 1 , the ratio of $\mathrm{A} 2-2$ decreases from $18 \%$, when at the same time the ratio of A2-5 increases from 19\%. These data are respectively $-34 \%$ and $+30 \%$ for trough concentrations in group 2 . The variations are important too for analogs A2-2, A2-3 and A2-4, ranging from $-21 \%$ to $+15 \%$.

In what concerns the peak concentrations, for both groups, the variations are less pronounced, but go in a same way for each of the analog type.

\section{Discussion}

As already stated, an accumulation of teicoplanin occurs in

\begin{tabular}{|c|c|c|c|}
\hline & & HPLC & FPIA \\
\hline & PEAKS & & \\
\hline \multirow{3}{*}{$\begin{array}{l}\text { group } 1 \\
(6 \mathrm{mg} / \mathrm{kg})\end{array}$} & $1^{\text {st }}$ dose & $32.8 \pm 7.0$ & $38.2 \pm 6.7$ (NS) \\
\hline & $3^{\text {rd }}$ dose & $46.0 \pm 8.8$ & $58.2 \pm 5.7$ (NS) \\
\hline & Last dose & $43.5 \pm 4.8$ & $\begin{array}{c}57.2 \pm 5.2(S) \\
p<0,002[55-60]\end{array}$ \\
\hline \multirow{4}{*}{$\begin{array}{c}\text { Group } 2 \\
\text { (12 then } 15 \text { mg/kg) }\end{array}$} & $1^{\text {st }}$ dose & $60.8 \pm 15.3$ & $71.4 \pm 19.3$ (NS) \\
\hline & $3^{\text {rd d }}$ dose & $106.2 \pm 17.0$ & $120.3 \pm 18.4(\mathrm{NS})$ \\
\hline & last dose & $92.5 \pm 11.4$ & $\begin{array}{c}121.2 \pm 15.4(\mathrm{~S}) \\
p<0,001[115-125]\end{array}$ \\
\hline & TROUGHS & & \\
\hline Group 1 (6 mg/kg) & Last dose & $16.1 \pm 1.25$ & $16.0 \pm 2.1(\mathrm{NS})$ \\
\hline $\begin{array}{c}\text { Group } 2(12 \text { then } 15 \\
\mathrm{mg} / \mathrm{kg})\end{array}$ & Last dose & $14.1 \pm 3.1$ & $\begin{array}{c}17.9 \pm 3.5(S) \\
p<0,002\end{array}$ \\
\hline
\end{tabular}

Table 1: Peak and trough concentrations in both groups as measured by HPLC and FPIA. 


\begin{tabular}{|c|c|c|c|c|}
\hline $\begin{array}{c}\text { GROUP } 1 \\
\left(\begin{array}{c}6 \mathrm{mg} / \mathrm{kg}, \mathrm{n}=8) \\
\text { troughs }\end{array}\right.\end{array}$ & & $\begin{array}{c}\text { Mean\% of total } \\
\text { teicoplanin for each } \\
\text { analog }\end{array}$ & S.D. & $\begin{array}{c}\text { Delta } \\
\text { D11 - D1 }\end{array}$ \\
\hline \multirow{2}{*}{ A2-1 } & D1 & 4.05 & 0.47 & \multirow{2}{*}{-18} \\
\hline & D11 & 3.32 & 0.43 & \\
\hline \multirow{2}{*}{ A2-2 } & D1 & 45.8 & 1.6 & \multirow{2}{*}{$-11 \%$} \\
\hline & D11 & 40.7 & 1.9 & \\
\hline \multirow{2}{*}{ A2-3 } & D1 & 7.7 & 1.1 & \multirow{2}{*}{$-21 \%$} \\
\hline & D11 & 6.1 & 1 & \\
\hline \multirow{2}{*}{ A2-4 } & D1 & 17.1 & 1 & \multirow{2}{*}{$+15 \%$} \\
\hline & D11 & 19.6 & 1.6 & \\
\hline \multirow{2}{*}{ A2-5 } & D1 & 25.3 & 1.3 & \multirow{2}{*}{$+19 \%$} \\
\hline & D11 & 30.3 & 0.6 & \\
\hline
\end{tabular}

Table 2: Teicoplanin trough concentrations at Day 1 (D1) and Day 11 (D11), for group 1 (upper) and group 2 (lower): percent of each analog of the A2 group, and delta between D11 and D1.

\begin{tabular}{|c|c|c|c|c|}
\hline $\begin{array}{c}\text { GROUP } 1 \\
\left(\begin{array}{c}6 \mathrm{mg} / \mathrm{kg}, \mathrm{n}=8) \\
\text { peaks }\end{array}\right.\end{array}$ & & $\begin{array}{c}\text { Mean } \% \text { of total } \\
\text { teicoplanin for each } \\
\text { analog }\end{array}$ & S.D. & $\begin{array}{c}\text { Delta } \\
\text { D11-D1 }\end{array}$ \\
\hline \multirow{2}{*}{ A2-1 } & D1 & 5.7 & 0.7 & \multirow{2}{*}{$-12 \%$} \\
\hline & D11 & 5.0 & 0.3 & \\
\hline \multirow{2}{*}{ A2-2 } & D1 & 50.5 & 1.0 & \multirow{2}{*}{$-5.3 \%$} \\
\hline & D11 & 47.8 & 0.7 & \\
\hline \multirow{2}{*}{ A2-3 } & D1 & 8.8 & 0.5 & \multirow{2}{*}{$-10.2 \%$} \\
\hline & D11 & 7.9 & 0.4 & \\
\hline \multirow{2}{*}{ A2-4 } & D1 & 16.0 & 0.8 & \multirow{2}{*}{$+7.5 \%$} \\
\hline & D11 & 17.2 & 0.8 & \\
\hline \multirow{2}{*}{ A2-5 } & D1 & 18.9 & 0.7 & \multirow{2}{*}{$+17 \%$} \\
\hline & D11 & 22.1 & 0.7 & \\
\hline
\end{tabular}

Table 3: Teicoplanin peak concentrations at Day 1 (D1) and Day 11 (D11), for group 1 (upper) and group 2 (lower): percent of each analog of the A2 group, and delta between $\mathrm{D} 11$ and D1.

plasma after repeated administration [11]. The various components of teicoplanin differ between themselves by their lipophilicity, as indicated, for example, by their differing behavior in reversed phase HPLC [10]. This accumulation phenomenon, due to differences in lipophilicity, may be expected to vary from one analog to the other during the time course of a treatment. These different lipophilicities, together with the resulting different protein binding, could make one anticipate the existence of differences in their pharmacokinetic behavior and profile. Thus, when lipophilicity increases, Bernaregi et al. [12] reported a decrease in the volume of distribution and the total clearance of teicoplanin. They anticipated an increased accumulation for the more lipophilic compounds during the treatment but also between the $24 \mathrm{~h}$ intervals separating two administrations.

We have evidenced this phenomenon in our work. There exist important differences in the evolution of the ratio to total A2teicoplanin of the different analogs from the A2 group. The more lipophilic compounds A2-3 to A2-5 increase and the less lipophilic A2-1 and A2-2 decrease. This occurs with all trough and peak samples of the 24 patients all along the study (data not shown) to finally reach the D11 minus D1 differences shown in Tables 2 and 3, either for peaks or for residuals. It also occurs in the interval of two administrations of teicoplanin between the peak and the residual for both dosage regimens. This difference is more pronounced for residuals when compared with peaks because the $24 \mathrm{~h}$ or $48 \mathrm{~h}$ elapsed time between two administrations allowed a significant concentration in blood of the more lipophilic compounds. For the peaks, the injection of "fresh" teicoplanin should have led to more or less "re-initialized" ratios of the different analogs and thus minimize the delta seen for the residuals.

The extend of variations of the A2 group analogs that may occur after teicoplanin administration are directly related to the initial ratio of the different analogs of the injected product. We have described here the variations resulting from the initial composition of the innovator (Targocid/Targosid). Up to now, there is no proof that the variations with time of the different analogs relative to the different marketed generics are equivalent, neither in blood nor in tissues. Colombo et al. [10] have reported that several teicoplanin generics products significantly differ in pharmaceutical quality from the originator on sub-group A2 distribution. The variations with time of the ratio of the various analogs of the $\mathrm{A} 2$ group seen in blood are likely to also exist in tissues. We have to keep in mind that the activities of the major components of teicoplanin against specific microbial species differ [11]. More, there is a synergistic activity of the different components of teicoplanin innovator in a certain ratio of these components [9]. Other ratios being different from those of innovator have not demonstrated to be synergic up to now.

Very recently, Rodriguez et al. [2] reported generic vancomycin to be less active than originator. More, it enriches resistant subpopulations of S.aureus after exposure in an animal model [16], as far as resistance is one of the natural consequences of suboptimal treatment of infection. This remains to be investigated with teicoplanin generics, as long as we do not know whether their analogs profiles are superimposable to those of innovator and have an equivalent tissue distribution and bactericidal activity.

Thus, as long as the effects of the differences of the A2 analogs have not been documented, concluding in bioequivalence of the different generics with the innovator is highly questionable.

Another matter of concern exits with the generics. The differences in concentrations obtained between FPIA and HPLC are well known $[17,18]$ and, as occurred in our study, are systematically in favor of FPIA that gives higher concentrations than HPLC. FPIA is an immunoassay that uses sheep antibodies. It is likely to occur that the antibodies may have different affinity for the different analogs of teicoplanin and be more or less specific for one or the other analog, or many of them. Consequently, a variation with time of the different ratios of the analogs may lead to a non-linearity of the antigen-antibody responses of the immunoassay and explain, at last partially, the differences seen between HPLC and FPIA. The demonstration of this non-linearity may only be noticeable when there is a threshold level of accumulation or decrease of one or more analogs.

Most of the laboratories monitor teicoplanin levels by FPIA. According to the differences seen in the composition of the various generics [10], for one given 'true' concentration of total teicoplanin that would have been measured by HPLC, there is a risk of obtaining varying results as a function of the generics employed, since the distribution of components unpredictably changes with producer. Thus this represents an additional risk of having various marketed generics, the compositions of which are not similar between themselves and differs from the originator.

In conclusion, the complex pharmacokinetic profiles of the A2 group analogs of teicoplanin innovator remain to be demonstrated to be the same in the numerous generics that differ in composition. If this would not be the case, the incidences on the tissue penetration ratio, the bactericidal effects in blood and tissues, the resistance risks, and potential risky consequences on therapeutic monitoring by FPIA make that the presently assumed bioequivalence between generics and innovator remains highly questionable.

This underscore the need of redefining the regulatory rules before 
Citation: Jehl F, Schramm F, Colombo P, Brink AJ (2013) Group A2 Analogs of Innovator Teicoplanin: Variability of their Ratio during Treatment Time Course in Human Blood; Consequences for the Generics. Pharmaceut Reg Affairs 2: 112. doi:10.4172/2167-7689.1000112

the marketing authorization is given to certain antibiotics, as it has been recently suggested [19].

\section{References}

1. Vesga O, Agudelo M, Salazar BE, Rodriguez CA, Zuluaga AF, et al. (2010) Generic vancomycin products fail in vivo despite being pharmaceutical equivalent of the innovator. Antimicrobial Agents Chemotherapy 54: 32713279 .

2. Rodriguez CA, Agudelo M, Cataño JC, Zuluaga AF, Vesga O (2009) Potential therapeutic failure of generic vancomycin in a liver transplant patient with MRSA peritonitis and bacteremia. J Infect 59: 277-280.

3. Fujimura S, Fuse K, Takane H, Nakano Y, Gomi K, et al. (2011) Antibacterial effects of brand-name teicoplanin and generic products against clinical isolates of methicillin-resistant Staphylococcus aureus. J Infect Chemother 17: 30-33.

4. Rodriguez CA, Agudelo M, Zuluaga AF, Vesga O (2010) In vitro and in vivo comparison of the anti-staphylococcal efficacy of generic products and the innovator of oxacillin. BMC Infect Dis.

5. Zuluaga AF, Agudelo M, Cardeño JJ, Rodriguez CA, Vesga O (2010) Determination of therapeutic equivalence of generic products of gentamicin in the neutropenic mouse thigh infection model. PLoS One 5.

6. Multisource (generic) pharmaceutical products: guidelines on registration requirements to establish interchangeability, World Health Organization 2005.

7. European Medicines Agency. Committee for medicinal products: guideline on the investigation of bioequivalence. 2010.

8. Taurino C, Frattini L, Marcone GL, Gastaldo L, Marinelli F (2011) Actinoplanes teichomyceticus ATCC 31121 as a cell factory for producing teicoplanin. Microbial Cell Factories 10: 82-95

9. European Medicines Agency. Assessment report. Review under Article $5(3)$ of regulation $(E C) n^{\circ} 726 / 2004$. TEICOPLANIN. Procedure $n^{\circ}$ : EMEA/ $\mathrm{H} / \mathrm{A}-5(3) / 1315$

10. Colombo G, Rossi A, Pala D, Buttini F, Colombo P. Complex product composition generates risks for generic substitution also with dosage forms with IV administration Expert Opinion in Drug Safety.

11. Hanada K, Kobayashi A, Okamori Y, Kimura T, Ogata H (2005) Improved quantitative determination of total and unbound concentrations of six teicoplanin components in human plasma by high performance liquid chromatography. Biol Pharm Bull 28: 2023-2025.

12. Bernareggi A, Danese A, Cometti A, Buniva G, Rowland M (1990) Pharmacokinetics of individual components of teicoplanin in man. J Pharmacokinet Biopharm 18: 525-543.

13. Rouveix B, Jehl F, Drugeon H, Brumpt I, Caulin E (2004) Randomized comparison of serum teicoplanin concentrations following daily or alternate daily dosing in healthy adults. Antimicrob Agents Chemother 48: 2394-2399.

14. Jehl F, Monteil H, Tarral A (1988) HPLC quantitation of the six main components of teicolanin in biological fluids. J Antimicrob Chemother 21: 53-59.

15. Schwartz $D(1983)$ Méthodes statistiques à l'usage des médecins et des biologistes. Flammarion Medecine Science eds.142-162.

16. Rodriguez CA, Agudelo M, Zuluaga AF, Vesga O (2012) Generic vancomycin enriches resistant subpopulations of Staphylococcus aureus after exposure in a neutropenic mouse thigh infection model. Antimicrob Agents Chemother 56: 243-247.

17. Awni WM, St Peter WL, Guay DRP, Kenny MT, Matzke GR (1991) Teicoplanin measurement in patients with renal failure: comparison of fluorescence polarization immunoassay, microbiological assay, and high performance liquid chromatographic assay. Ther Drug Monit 13: 511-517.

18. Bourget P, Lesne-Hulin A, Sertin A, Maillot A, Alaya M, et al. (1997) Fluorescence polarization immunoassay: Does it always represent a reliable method to monitor treatment with teicoplanin?: Comparison with data obtained by high-performance liquid chromatography International Journal of Pharmaceutics 146: 167-174.

19. Kirkpatrick CJ, Rangoonwala R, Reshetnikov M, Barbeck M, Ghanaati S (2013) Non equivalence of antibiotics generic drugs and risk for Intensive care patients. Pharmaceut Reg Affairs 2: 109. 